\title{
Effect of gene order in DNA constructs on gene expression upon integration into plant genome
}

\author{
M. Aydın Akbudak ${ }^{1} \cdot$ Vibha Srivastava $a^{2,3}$
}

Received: 28 November 2016/Accepted: 29 January 2017/Published online: 29 May 2017

(C) Springer-Verlag Berlin Heidelberg 2017

\begin{abstract}
Several plant biotechnology applications are based on the expression of multiple genes located on a single transformation vector. The principles of stable expression of foreign genes in plant cells include integration of full-length gene fragments consisting of promoter and transcription terminator sequences, and avoiding converging orientation of the gene transcriptional direction. Therefore, investigators usually generate constructs in which genes are assembled in the same orientation. However, no specific information is available on the effect of the order in which genes should be assembled in the construct to support optimum expression of each gene upon integration in the genome. While many factors, including genomic position and the integration structure, could affect gene expression, the investigators judiciously design DNA constructs to avoid glitches. However, the gene order in a multigene assembly remains an open question. This study addressed the effect of gene order in the DNA construct on gene expression in rice using a simple design of two genes placed in two possible orders with respect to the genomic context. Transgenic rice lines containing green fluorescent protein (GFP) and $\beta$-glucuronidase (GUS) genes in two distinct orders were developed by Cre-lox-mediated sitespecific integration. Gene expression analysis of transgenic lines showed that both genes were expressed at similar
\end{abstract}

M. Aydın Akbudak

akbudak@akdeniz.edu.tr

1 Department of Agricultural Biotechnology, Akdeniz University, Antalya 07059, Turkey

2 Department of Crop, Soil and Environmental Sciences, University of Arkansas, Fayetteville, AR 72701, USA

3 Department of Horticulture, University of Arkansas, Fayetteville, AR 72701, USA levels in either orientation, and different transgenic lines expressed each gene within $1-2 \times$ range. Thus, no significant effect of the gene order on gene expression was found in the transformed rice lines containing precise site-specific integrations and stable gene expression in plant cells could be obtained with altered gene orders. Therefore, gene orientation and integration structures are more important factors governing gene expression than gene orders in the genomic context.

Keywords Transgene expression - Multigene transformation - Gene order - Gene orientation - Sitespecific integration $\cdot$ Cre-lox

\section{Introduction}

Plant cell transformation involves integration of single or multiple gene(s) to express the encoded proteins. Designing DNA constructs consisting of multiple genes is fundamentally tied to the success of plant transformation projects. Multiple genes could be assembled in a single transformation vector for transfer into plant cells. When assembling two or more genes, investigators face the question of the order in which the genes should be assembled to obtain optimum expression upon integration into plant cells. This basic part of the construct design is guided by the principles of stable gene expression in transformed plant cells as the goal of the experiment is mostly to express the gene(s) at high levels. It is well established that a full-length single-copy integration is correlated with high expression levels (Akbudak et al. 2010; Chawla et al. 2006; Srivastava and Ow 2002). Furthermore, tail-to-tail orientation, i.e., two genes having converging transcriptional direction are not desired as 
antisense transcripts could be generated through the readthrough transcription mechanisms leading possibly to silencing of both genes (Luo and Chen 2007; Nicholson and Srivastava 2009; Ulker et al. 2008). A recent paper showed that diverging orientation of genes in which the promoters of the genes are juxtaposed; expression of each gene could be influenced (Qiu et al. 2016), possibly due to the proximity of their enhancers. Therefore, in a multigene construct, all genes should be placed in the same transcriptional orientation (Akbudak et al. 2010; Svitashev et al. 2002). However, whether the order of genes in the assembly also affects gene expression has not been clearly investigated.

When combining genes in a binary vector, the gene order is determined by the T-DNA borders, right border (RB) and left border (LB), since the transfer of T-DNA starts at RB, and most T-DNA fragments are intact at RB but contain variable lengths at the LB end. The significance of gene order would be difficult to determine on non-binary vectors delivered by gene gun, since the whole vector is scrambled into the genome and imprecisely integrated, generating integration structures that alter the gene order designed in the vector (Makarevitch et al. 2003). However, gene gun delivery of minimal cassettes (DNA constructs without the vector backbones) achieves a higher rate of single-copy integration similar to that observed in Agrobacterium-mediated T-DNA delivery (Fu et al. 2000; Jackson et al. 2013).

Furthermore, methods of precise gene integrations are also available that target the delivered DNA to specific sites in the genome, generating predictable integration structures. These methods rely on DNA recombination (site-specific recombination or homologous recombination) to achieve site-specific integration (Albert et al. 1995; Srivastava et al. 2004; Terada et al. 2002). Recombinasemediated site-specific integration using Cre-lox and FLPFRT systems is highly efficient (Albert et al. 1995; Day et al. 2000; Kilby et al. 1995; Li et al. 2010; Lloyd and Davis 1994; Srivastava et al. 2004); however, it requires placing a recombination site (lox or FRT) into plant genomes first. Homologous recombination-based gene targeting does not require placement of a recombination sites; however, its observed efficiency in higher plants is much lower even with the newly developed gene editing reagents that induce targeted double-stranded breaks (DSB) that, in turn, trigger homologous recombination (Puchta et al. 1993; Steinert et al. 2016). The newly developed gene targeting and genome editing technologies which make use of site-specific nucleases to induce homologous recombination of delivered DNA do not require such pre-integrated DNAs (Cermak et al. 2011; Feng et al. 2013; Townsend et al. 2009).
Gene stacking or multigenic transformations either by non-targeted approaches (minimal cassettes and T-DNA) or by site-specific integration are important biotechnological tools for engineering useful traits in plants or producing pharmaceutical proteins or metabolites among other applications (Srivastava and Thomson 2016). In all of these approaches, the gene order in the DNA construct is a highly relevant question, which should be experimentally addressed. This study determined the expression of two genes placed in two different orders in the DNA construct relative to the selection marker gene, and determined gene expression upon site-specific integration of these constructs into the rice genome. Site-specific integration conserves gene order determined in the construct upon its integration into plant cell and removes the gene expression variation caused by the genomic positions (position-effect variation) (Albert et al. 1995; Chawla et al. 2006).

In this study, a simple experimental design of rice transformation with two different constructs containing distinct gene orders of green fluorescent protein $(G F P)$ and $\beta$-glucuronidase $(G U S)$ genes was used. No significant effect of the gene order in the tested genomic context on gene expression was found. While different genomic contexts could cast different effects on gene expression, it is likely that the effect would be predictably uniform on all genes. Therefore, this study suggests that investigators could develop DNA constructs containing variable gene orders for multigene transformation and expect predicted expression of each gene regardless of the order in which they are assembled as long as the relative orientation of genes in the assembly is the same.

\section{Results and discussion}

\section{Molecular design}

The gene order effect was analyzed through site-specific integration of GFP and GUS genes placed in two possible orders at the T5 locus through Cre-lox-mediated sitespecific integration (Fig. 1a). Site-specific integration approach (Srivastava et al. 2004) was used to avoid the gene expression variations caused by genomic position and integration structures. T5 locus contains a single copy of T-DNA, as shown in Fig. 1a, that contains lox76 site for site-specific integration via Cre-lox recombination (Akbudak and Srivastava 2011; Srivastava et al. 2004; Srivastava and Ow 2002). The T-DNA contains a cre gene driven by maize ubiquitin promoter (ZmUbi1) with lox75 site placed between the promoter and the cre coding sequence. Sitespecific integration into lox76 will generate an integration structure with a fixed gene orientation and gene order. Two 
(a)

(b)

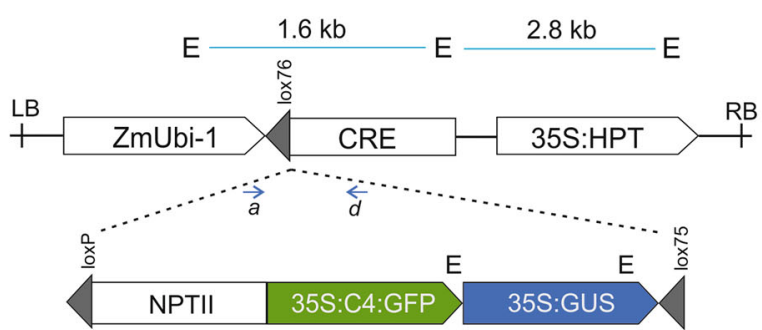

or

(c)

pAA13

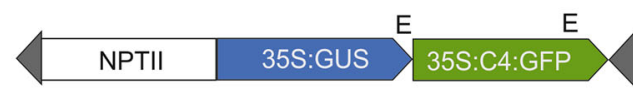

(d)

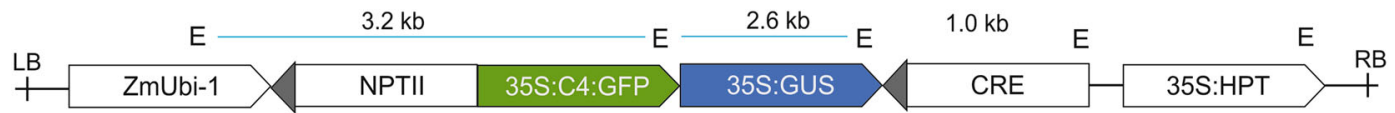

(e)

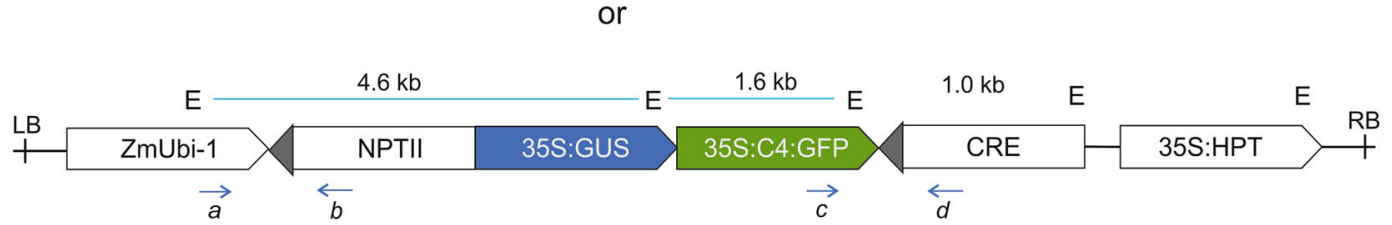

Fig. 1 Molecular strategy of site-specific integration (SSI). a Structure of T-DNA used to generate the target site in rice genome (Taipei309) and termed as $T 5$ site. The $T 5$ site contains a single-copy of the T-DNA that contains lox 76 target site (gray triangle) and Cre activity. It also contains $35 \mathrm{~S}: \mathrm{HPT}$ as the selection marker genes. b, c Structures of the two donor vectors, pAA12 and pAA13, designed for sitespecific integration into $T 5$ site. Each contains two lox sites (gray triangles), $\operatorname{lox} P$ at $5^{\prime}$ end and $\operatorname{lox} 75$ at $3^{\prime}$ end, for Cre-lox-mediated site-specific integration into T5 site via lox $75 \times$ lox 76 recombination. The two vectors contain a promoter-less marker gene, NPT II, for the selection of site-specific integration (SSI) and distinct gene orders of

donor constructs, pAA12 and pAA13, were developed that contain promoter-less NPTII gene and the genes-of-interest, GFP and GUS, cloned in two possible orders, GFPGUS or GUS-GFP (Fig. 1b, c). Site-specific integration (SSI) of these constructs would generate integration structures that differ only in the gene order of two genes without any predictable change in the genomic context or gene orientation. All SSI lines derived from pAA12 contain $G F P-G U S$ cassettes, while those from pAA13 contain the reverse order, i.e., GUS-GFP (Fig. 1d, e). Both pAA12 and pAA13 contain identical regulatory elements for each gene, i.e., 35S:C4 promoter for $G F P$ and $35 \mathrm{~S}$ promoter for $G U S$ and $n o s 3^{\prime}$ for transcription termination of each gene. The SSI lines developed by gene gun delivery of pAA12 or pAA13 into T5 callus were characterized by PCR and Southern blot and subjected to gene expression analysis by fluorescence or GUS assay.
GFP and GUS genes as shown. d, e Integration of the donor constructs via Cre-lox recombination into the target site generates a predictable SSI structure. pAA12 lines contain GFP-GUS order, while pAA13 contain GUS-GFP order within the T5 locus relative to the LB and RB (T-DNA left and right borders). ZmUbil maize ubiquitin-1 promoter, $H P T$ hygromycin phosphotransferase gene, $35 S$ cauliflower mosaic virus 35S RNA promoter, $C 4$ maize PPDK promoter, NPT neomycin phosphotransferase gene. Each gene carries a nopaline synthase $3^{\prime}$ transcriptional termination signal (not shown). PCR primer (a-d) sites and their orientation (blue arrows), and EcoRI (E) sites are indicated

\section{Characterization of transgenic callus lines}

Two different experiments involving bombardment of T5 callus with either pAA12 or pAA13 generated a number of geneticin-resistant lines. Each line was analyzed by PCR using primers $a-b$ and $c-d$ to determine the presence of predicted SSI junctions. In addition, primer pair $a-d$ was used to determine biallelic/monoallelic integrations occurred (Fig. 1a, e). PCR analysis revealed that 23 pAA12 (GFPGUS) lines and 7 pAA13 (GUS-GFP) lines carried the expected SSI junctions. The rest of the lines were removed from further analysis. The PCR for $a-d$ junction indicated monoallelic integration in all except one pAA12 line, which contained biallelic integration. The presence of SSI junctions indicates precise integration of the construct into $T 5$ locus, and the absence of the parental $a-d$ junction indicates biallelic integration. A representative PCR data is shown in Fig. 2a. 
(a)

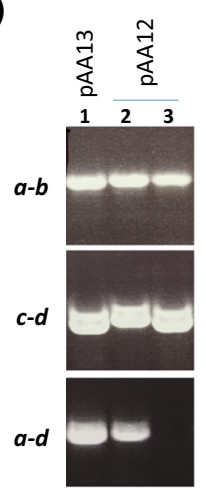

(b)

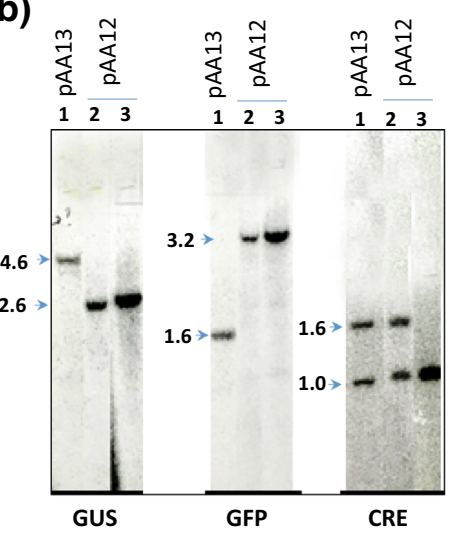

Fig. 2 Molecular analysis of transgenic lines. a PCR analysis of SSI lines obtained by transformation of T5 callus with pAA12 or pAA13 using primer pairs indicated on each panel, and mapped on Fig. 1. b Southern analysis of SSI lines. Each panel show SSI fragments with GUS, GFP, or CRE probes from EcoRI-digested genomic DNA of (see Fig. 1d, e). These fragment sizes match the predicted sizes EcoRI fragment sizes of SSI locus (Fig. 1d, e). Lane 1 in each panel represents pAA13 line 13.6 (monoallelic), and lanes 2 and 3 represent pAA12 lines, 12.15 (monoallelic) and 12.11 (biallelic), respectively

Subsequently, DNA blot analyses on EcoRI-digested genomic DNA were carried out to confirm the presence of SSI structure, and determine the presence of additional

copies of the vectors. Representative Southern blots are given in Fig. 2b. The putative SSI lines could contain random integrations of the vector DNA since 'illegitimate' recombination cannot be suppressed. Fourteen out of twenty-three pAA12 lines and six out of seven pAA13 lines showed the predicted hybridization pattern with GUS, GFP, and Cre probes (Fig. 2b). These lines were designated as precise-SSI lines. However, one line each of pAA12 and pAA13 showed an extra band with GUS or GFP probe, indicating the presence of an extra copy (data not shown) (Table 1). The remaining lines showed complex integration pattern indicating the presence of numerous extra copies (data not shown). Of these complex-SSI lines, two complex-SSI of pAA12 and the single complexSSI of pAA13 were included for expression analysis.

The attempts to regenerate these callus lines failed; however, most lines were maintained for up to 1 year by transferring to fresh selection plates regularly. To assess the potential problem of chimerism, defined as contamination of 'untransformed' target cells, we used a molecular approach of evaluating relative intensities of cre gene on Southern blot or PCR. Hybridization with cre probe on a Southern blot displays two distinct bands representing either SSI site $(1.0 \mathrm{~kb})$ or target site $(1.6 \mathrm{~kb})$. Both 1.0 and

Table 1 Characterization of transgenic lines and expression analyses

\begin{tabular}{|c|c|c|c|c|c|c|c|c|c|}
\hline \multirow[t]{2}{*}{ Vector } & \multirow[t]{2}{*}{ Line } & \multirow[t]{2}{*}{ Integration pattern } & \multicolumn{2}{|c|}{ Copy \# } & \multicolumn{3}{|c|}{ PCR } & \multirow[t]{2}{*}{$\mathrm{GFP}^{\mathrm{a}}$ ( $\mu \mathrm{g} / \mathrm{mg}$ total protein) } & \multirow[t]{2}{*}{$\mathrm{GUS}^{\mathrm{a}}$ (nmole/min/mg) } \\
\hline & & & GFP & GUS & $\mathrm{a}-\mathrm{b}$ & $\mathrm{c}-\mathrm{d}$ & $a-d$ & & \\
\hline \multirow[t]{15}{*}{ pAA12 } & 12.1 & Precise & 1 & 1 & + & + & + & 2.70 & 27.13 \\
\hline & 12.5 & Precise & 1 & 1 & + & + & + & 2.78 & 21.44 \\
\hline & 12.8 & Precise & 1 & 1 & + & + & + & 2.76 & 37.81 \\
\hline & 12.11 & Precise & 2 & 2 & + & + & - & 6.61 & 47.86 \\
\hline & 12.12 & Precise & 1 & 1 & + & + & + & 2.51 & 22.80 \\
\hline & 12.13 & Precise & 1 & 1 & + & + & + & 3.29 & 26.60 \\
\hline & 12.15 & Precise & 1 & 1 & + & + & + & 2.86 & 40.29 \\
\hline & 12.16 & Precise & 1 & 1 & + & + & + & 2.29 & 25.08 \\
\hline & 12.17 & Precise & 1 & 1 & + & + & + & 4.04 & 31.67 \\
\hline & 12.26 & Precise & 1 & 2 & + & + & + & 3.05 & 25.13 \\
\hline & 12.30 & Precise & 1 & 1 & + & + & + & 2.38 & 24.43 \\
\hline & 12.32 & Precise & 1 & 1 & + & + & + & 3.31 & 21.99 \\
\hline & 12.33 & Precise & 1 & 1 & + & + & + & 2.32 & 37.42 \\
\hline & 12.24 & Complex & $>4$ & 2 & + & + & + & 3.62 & 6.21 \\
\hline & 12.35 & Complex & $>2$ & $>2$ & + & + & + & 4.03 & 9.16 \\
\hline \multirow[t]{7}{*}{ pAA13 } & 13.2 & Precise & 2 & 1 & + & + & + & 4.85 & 23.27 \\
\hline & 13.6 & Precise & 1 & 1 & + & + & + & 2.77 & 25.43 \\
\hline & 13.9 & Precise & 1 & 1 & + & + & + & 2.56 & 25.10 \\
\hline & 13.13 & Precise & 1 & 1 & + & + & + & 2.64 & 23.75 \\
\hline & 13.20 & Precise & 1 & 1 & + & + & + & 2.74 & 23.11 \\
\hline & 13.21 & Precise & 1 & 1 & + & + & + & 2.61 & 22.14 \\
\hline & 13.8 & Complex & $>5$ & $>4$ & + & + & + & 2.22 & 6.14 \\
\hline
\end{tabular}

${ }^{\text {a }}$ Standard deviation of mean (sdom; $n=3$ ) in GFP amount and GUS activity were depicted as error bars in Fig. 3 

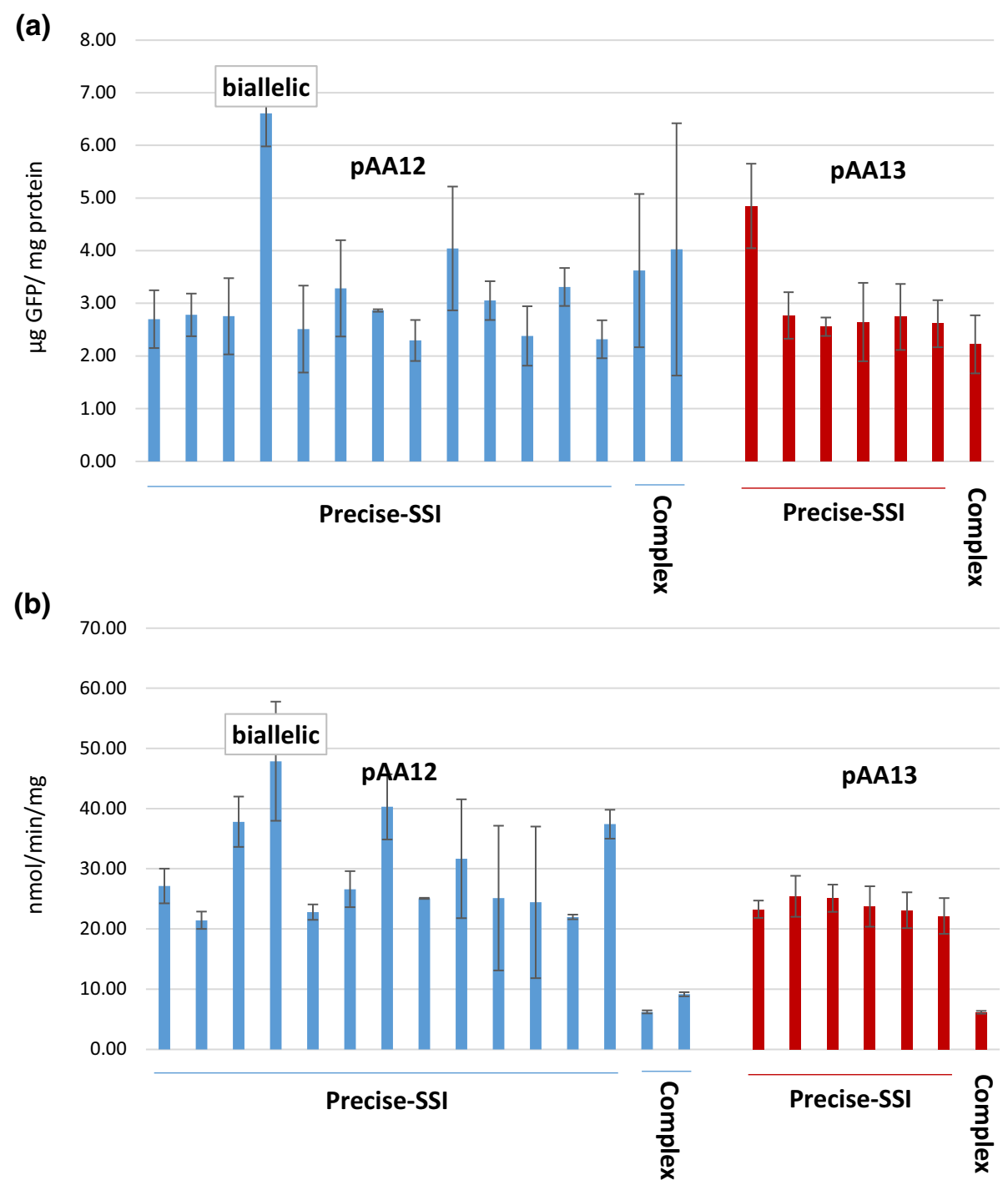

Fig. 3 Effect of gene order on gene expression: a GFP and b GUS expression levels in pAA12 (blue bars) and pAA13 (red bars) SSI lines containing the predictable SSI only (precise) or the extra copies of the plasmids in addition to the predictable SSI (complex). Error bars represent standard deviation

$1.6 \mathrm{~kb}$ bands are expected from monoallelic SSI, whereas $1.0 \mathrm{~kb}$ band would be produced from biallelic SSI (see Fig. 1). Equal intensity of 1.0 and $1.6 \mathrm{~kb}$ bands in monoallelic lines suggests the absence of contamination by untransformed target cells (Fig. 2b). In the biallelic SSI line, target site-specific PCR (primers $a-d$ ) was conducted to rule out the contamination (Figs. 1a, 2a). These analyses suggested that all SSI lines were homogenous as contamination of untransformed T5 cells was undetectable. Furthermore, PCR and Southern analyses confirmed that all transgenic lines contained site-specific integrations of pAA12 or pAA13 constructs, which rules out the speculation that transgene expression differences could occur due to genomic position or integration structure variations in the precise-SSI lines.

\section{Transgene expression analysis}

Fifteen pAA12 and seven pAA13 lines were subjected to GUS and GFP expression analysis (Table 1). Three of these lines, two pAA12 and one pAA13 line, were complex-SSI, while the remaining were precise-SSI containing the predictable structures, as shown in Fig. 1. The GUS activity was assessed using histochemical staining and MUG assay, whereas GFP was quantified by fluorescence spectrometry. All lines stained positive for GUS activity and showed green fluorescence, indicating the expression of GUS and GFP genes.

The quantitative measurement of GUS activity by MUG assay indicated high expression in precise-SSI lines, whereas a significant suppression in the complex-SSI lines. 
In contrast, GFP gene highly expressed in both precise-SSI and complex-SSI lines (Table 1; Fig. 3a, b). This observation could be an artefact based on the functional difference of the two proteins (enzyme vs fluorescent protein) and analytical methods used for their measurements (enzymatic assay vs fluorescence). Higher fluctuations in GUS activity as compared to GFP activity in transgenic rice were also reported by Akbudak et al. (2010). In general, gene suppression could occur in multi-copy lines even if one of the copies represent precise-SSI (Srivastava et al. 2004), and the molecular basis of suppression is most likely RNAi, since segregation of the precise-SSI results in restoration of high gene activity (Chawla et al. 2006).

Furthermore, one of the SSI lines, 12.11, contained biallelic integration of pAA 12 construct. Accordingly, this line expressed highest levels of GFP and GUS activities (Table 1; Fig. 3a, b). Similarly, line 13.2 that contained an extra copy of the GFP gene expressed highest levels of GFP among pAA13 lines. These two lines, therefore, display additive effects of gene copy number. The remaining precise-SSI lines expressed GFP and GUS genes within $1-2 \times$ variability (Table 1; Fig. 3a, b). Furthermore, the GFP or GUS expression ranges were similar in SSI lines regardless of the gene order, with the biallelic line, 12.11, showing $\geq 2 \times$ GFP expression level. The GFP expression level in precise-SSI lines was similar to that of complexSSI, suggesting lack of additive or suppressive effects of the extra copies on GFP gene expression. The GUS expression variation was also minimal ranging between 1 and $2 \times$ among SSI lines. These observations match with the previous report that found $2-3 \times$ variability in GUS expression among SSI lines carrying a single-copy of the GUS gene (Srivastava et al. 2004).

Next, all monoallelic lines were used to calculate average GFP or GUS expression produced by SSI lines carrying distinct gene orders of the two genes. This analysis showed that pAA12 and pAA13 constructs generated similar levels of gene expression of GFP or GUS genes from the T5 locus (Fig. 4). In summary, no significant effect of the gene order in the DNA construct was observed on gene expression and stable gene expression was obtained by both constructs. Therefore, genes could be assembled in any order to obtain optimal expression from a given genomic site provided that they contain full-length single-copy integrations.

\section{Materials and methods}

\section{Vector construction}

The donor vectors were generated in the backbone of pVS55 described by Srivastava et al. (2004). pVS55 (pUC18 backbone) contains lox sites (gray triangles) for

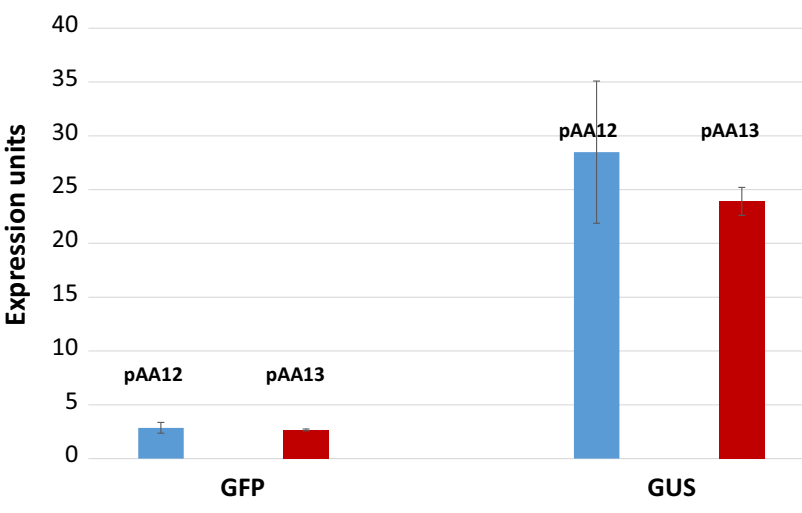

Fig. 4 Effect of gene order: average GFP and GUS expression values obtained from precise-SSI lines of pAA12 and pAA13. Expression units represent GFP and GUS units, as shown in Fig. 3, and error bars represent standard deviation

Cre-lox-mediated site-specific integration into plant genomes. pVS55 was cut with HindIII to replace the GUS gene with SpeI linker. The XbaI fragments consisting of 35S:C4:GFP and 35S:GUS cassettes were ligated into the SpeI, sequentially, to generate pAA12 and pAA13.

\section{Rice transformation}

Rice transformation was done by particle bombardment method using PDS 1000 gene gun (Bio-Rad, Inc.). Rice tissue culture media preparation was essentially following the method described by Hiei et al. (1994). The rice line T5 (Taipei-309) containing a target site, as depicted in Fig. 1a, was used for transformation. The transformation of T5 callus with pAA12 and pAA13 was done according to the protocol described by Srivastava (2013). The bombarded calli were selected on $100 \mathrm{mg} / \mathrm{l}$ Geneticin (Gibco BRL, Grand Island, NY, USA) and maintained by regular subcultures onto fresh media plates containing Geneticin.

\section{Molecular analysis}

Polymerase chain reaction (PCR) was carried out on the genomic DNA with primers 'a' (5'-TCTACTTCTGTTC ATGTTTGTG-3'), 'b’ (5'-CTCGATGCGATGTTTCG CTT-3'), 'c' (5'-GATTAGAGTCCCGCAATTAT-3'), and 'd' (5'-CTAATCGCCATCTTCCAGCA-3'). Taq Polymerase (Promega, Madison, WI) was used for all amplifications following the manufacturer's recommendation. The reactions consisted of 39 cycles of melting at $96{ }^{\circ} \mathrm{C}$ for $1 \mathrm{~min}$, annealing at $58{ }^{\circ} \mathrm{C}$ for $1 \mathrm{~min}$, and extension at $72{ }^{\circ} \mathrm{C}$ for $1 \mathrm{~min}$, followed by final extension for $7 \mathrm{~min}$ at $72{ }^{\circ} \mathrm{C}$. Southern blot analysis was carried out with three ${ }^{32}$ P-labeled DNA probes (GFP, GUS, and CRE). Approximately $5 \mu \mathrm{g}$ of genomic DNA digested with EcoRI, fractionated on $0.8 \%$ agarose gel, blotted on nylon membrane, 
was hybridized with the probes using standard Southern hybridization methods.

\section{Expression analysis}

GUS activity in calli was detected by the method described by Jefferson (1987). Callus pieces were submerged in the GUS staining solution containing $1 \mathrm{mM} \mathrm{X-Gluc} \mathrm{(Gold}$ Biotechnologies, St. Louis, MO, USA) and incubated at $37^{\circ} \mathrm{C}$. The quantitative measurement of GUS activity was done using the FluorAce GUS reporter kit (Bio-Rad, Inc.). Total protein of plant extracts was measured using the DC protein assay kit (Bio-Rad Inc.). A standard curve prepared with the dilution series of 4-methylumbelliferone (4-MU) was used to calculate GUS activity. A unit of GUS activity is defined as nmol 4-MU produced per minute from each milligram of the soluble protein $(\mathrm{nmol} / \mathrm{min} / \mathrm{mg})$. The GFP expression was observed visually under blue light using yellow filter. For the quantitative measurement of GFP, callus was ground in the extraction buffer $(10 \mathrm{mM}$ TrisEDTA, pH 8.0) at $4{ }^{\circ} \mathrm{C}$ and centrifuged at $13,000 \mathrm{rpm}$ for $20 \mathrm{~min}$ to collect the supernatant. Protein concentrations of the extracts were determined using the DC protein assay kit (Bio-Rad Inc.). GFP quantification was done using VersaFluor fluorometer (Bio-Rad Inc.) fitted with a $490 \pm 5 \mathrm{~nm}$ excitation filter and a $510 \pm 5 \mathrm{~nm}$ emission filter. A dilution series $(0.1-1 \mathrm{mg} / \mathrm{ml})$ of purified rGFPS65T protein (Clontech Inc., San Diego, CA, USA) was made in the extraction buffer to generate the standard curve. A unit of GFP is defined as microgram GFP per mg soluble protein $(\mu \mathrm{g} / \mathrm{mg}$ total protein)

Acknowledgements This research was supported by ABI grants and USDA-NIFA award 2014-38821-22460 to V.S. Authors are grateful to Y. Niwa for providing 35S:C4:GFP construct (GenBank Accession \# EF 090408.1).

\section{Compliance with ethical standards}

Conflict of interest Authors do not declare any conflict of interest.

\section{References}

Akbudak MA, Srivastava V (2011) Improved FLP recombinase, FLPe, efficiently removes marker gene from transgene locus developed by Cre-lox mediated site-specific gene integration in rice. Mol Biotechnol 49:82-89

Akbudak MA, More AB, Nandy S, Srivastava V (2010) Dosagedependent gene expression from direct repeat locus in rice developed by site-specific gene integration. Mol Biotechnol 45(1):15-23

Albert H, Dale EC, Lee E, Ow DW (1995) Site-specific integration of DNA into wild-type and mutant lox sites placed in the plant genome. Plant J 7(4):649-659

Cermak T, Doyle EL, Christian M, Wang L, Zhang Y, Schmidt C, Baller JA, Somia NV, Bogdanove AJ, Voytas DF (2011)
Efficient design and assembly of custom TALEN and other TAL effector-based constructs for DNA targeting. Nucl Acid Res 39(12):e82

Chawla R, Ariza-Nieto M, Wilson AJ, Moore SK, Srivastava V (2006) Transgene expression produced by biolistic-mediated, site-specific gene integration is consistently inherited by the subsequent generations. Plant Biotechnol J 4(2):209-218

Day CD, Lee E, Kobayashi T, Holappa LD, Albert H, Ow DW (2000) Transgene integration into the same chromosome location can produce alleles that express at a predictable level, or alleles that are differentially silenced. Gene Dev 14(22):2869-2880

Feng Z, Zhang B, Ding W, Liu X, Yang DL, Wei P, Cao F, Zhu S, Zhang F, Mao Y, Zhu JK (2013) Efficient genome editing in plants using a CRISPR/Cas system. Cell Res 23(10):1229-1232

Fu X, Duc LT, Fontana S, Bong BB, Tinjuangjun P, Sudhakar D, Twyman RM, Christou P, Kohli A (2000) Linear transgene constructs lacking vector backbone sequences generate lowcopy-number transgenic plants with simple integration patterns. Transgenic Res 9(1):11-19

Hiei Y, Ohta S, Komari T, Kumashiro T (1994) Efficient transformation of rice (Oryza sativa L.) mediated by Agrobacterium and sequence analysis of the boundaries of the T-DNA. Plant $\mathrm{J}$ 6:271-282

Jackson MA, Anderson DJ, Birch RG (2013) Comparison of Agrobacterium and particle bombardment using whole plasmid or minimal cassette for production of high-expressing, low-copy transgenic plants. Transgenic Res 22(1):143-151

Jefferson RA (1987) Assaying chimeric genes in plants: the GUS gene fusion system. Plant Mol Biol Rep 5(4):387-405

Kilby NJ, Davies GJ, Snaith MR, Murray JAH (1995) FLP recombinase in transgenic plants-constitutive activity in stably transformed tobacco and generation of marked cell clones in Arabidopsis. Plant J 8(5):637-652

Li B, Li N, Duan XG, Wei AY, Yang AF, Zhang JR (2010) Generation of marker-free transgenic maize with improved salt tolerance using the FLP/FRT recombination system. J Biotechnol 145(2):206-213

Lloyd AM, Davis RW (1994) Functional expression of the yeast FLP/ FRT Site-specific recombination system in Nicotiana tabacum. Mol Gen Genet 242(6):653-657

Luo ZH, Chen ZX (2007) Improperly terminated, unpolyadenylated mRNA of sense transgenes is targeted by RDR6-mediated RNA silencing in Arabidopsis. Plant Cell 19(3):943-958

Makarevitch I, Svitashev SK, Somers DA (2003) Complete sequence analysis of transgene loci from plants transformed via microprojectile bombardment. Plant Mol Biol 52(2):421-432

Nicholson SJ, Srivastava V (2009) Transgene constructs lacking transcription termination signal induce efficient silencing of endogenous targets in Arabidopsis. Mol Genet Genom 282:319-328

Puchta H, Dujon B, Hohn B (1993) Homologous recombination in plant cells is enhanced by in vivo induction of double-strand breaks into DNA by a site-specific endonuclease. Nucl Acid Res 21(22):5034-5040

Qiu T, Dong Y, Ren Y, Wang J, Yang M, Zhang J (2016) Effects of the sequence and orientation of an expression cassette in tobacco transformed by dual $B t$ genes. Plasmid $89: 1-8$

Srivastava V (2013) Site-specific gene integration in rice. Methods Mol Biol 956:83-93

Srivastava V, Ow DW (2002) Biolistic mediated site-specific integration in rice. Mol Breed 8(4):345-350

Srivastava V, Thomson J (2016) Gene stacking by recombinases. Plant Biotechnol J 14(2):471-482

Srivastava V, Ariza-Nieto M, Wilson AJ (2004) Cre-mediated sitespecific gene integration for consistent transgene expression in rice. Plant Biotechnol J 2(2):169-179 
Steinert J, Schiml S, Puchta H (2016) Homology-based double-strand break-induced genome engineering in plants. Plant Cell Rep 35:1429-1438

Svitashev SK, Pawlowski WP, Makarevitch I, Plank DW, Somers DA (2002) Complex transgene locus structures implicate multiple mechanisms for plant transgene rearrangement. Plant $\mathbf{J}$ 32(4):433-445

Terada R, Urawa H, Inagaki Y, Tsugane K, Iida S (2002) Efficient gene targeting by homologous recombination in rice. Nat Biotechnol 20(10):1030-1034
Townsend JA, Wright DA, Winfrey RJ, Fu FL, Maeder ML, Joung JK, Voytas DF (2009) High-frequency modification of plant genes using engineered zinc-finger nucleases. Nature 459:442-445

Ulker B, Peiter E, Dixon DP, Moffat C, Capper R, Bouche N, Edwards R, Sanders D, Knight H, Knight MR (2008) Getting the most out of publicly available T-DNA insertion lines. Plant J 56(4):665-677 